\title{
TRELLISES FORMED BY STABLE AND UNSTABLE MANIFOLDS IN THE PLANE
}

BY

\author{
ROBERT W. EASTON
}

\begin{abstract}
A trellis is the figure formed by the stable and unstable manifolds of a hyperbolic periodic point of a diffeomorphism of a 2-manifold. This paper describes and classifies some trellises. The set of homoclinic points is linearly ordered as a subset of the stable manifold and again as a subset of the unstable manifold. Each homoclinic point is assigned a type number which is constant on its orbit. Combinatorial properties of trellises are studied using type numbers and the pair of linear orderings. Trellises are important because their closures in some cases are strange attractors and in other cases are ergodic zones.
\end{abstract}

Introduction. In Chapter 33 of New Methods of Celestial Mechanics, Poincaré describes the figure formed by the stable and unstable manifolds of a hyperbolic fixed point of a transformation of the upper half plane as follows: "When we try to represent the figure formed by these two curves and their intersections, each of which corresponds to a doubly asymptotic solution, these intersections form a type of trellis, tissue, or grid with infinitely serrated mesh. Neither of the two curves must ever cut across itself again, but must bend back upon itself in a very complex manner in order to cut across all of the meshes in the grid an infinite number of times. The complexity of this figure will be striking, and I shall not even try to draw it. Nothing is more suitable for providing us with an idea of the complex nature of the three body problem, and of all the problems of dynamics in general...."

The aim of this paper is to understand figures of the type Poincaré described so well. If $p$ is a hyperbolic periodic point of a diffeomorphism of a 2-manifold, then the figure formed by its stable and unstable manifolds will be called the trellis of $p$ whenever these manifolds have nonempty intersection. Figure 1 illustrates the beginning development of such a trellis.

Similarly the figure formed by the stable and unstable manifolds of a cycle of hyperbolic periodic points is called the trellis of the cycle. A cycle of hyperbolic periodic points of a diffeomorphism is a finite collection $\underline{P}$ of periodic points having a cyclic permutation $\pi: \underline{P} \rightarrow \underline{P}$ such that the unstable manifold of $p$ intersects the stable manifold of $\pi(p)$ for each $p \in \underline{P}$. It is natural to conjecture that in some cases "ergodic zones" are the closures of trellises. Anosov transformations of the torus provide examples where this is the case $[\mathbf{1}]$. One may also conjecture that "strange attractors" are sometimes the closures of the unstable manifolds of cycles of hyperbolic periodic points. For certain values of the parameters $a$ and $b$ the Henon Map of the plane defined by $T(x, y)=(1+y-a x, b y)$ may provide examples.

Received by the editors May 7, 1985.

1980 Mathematics Subject Classification. Primary 58F13; Secondary 58F05, 58F11. 


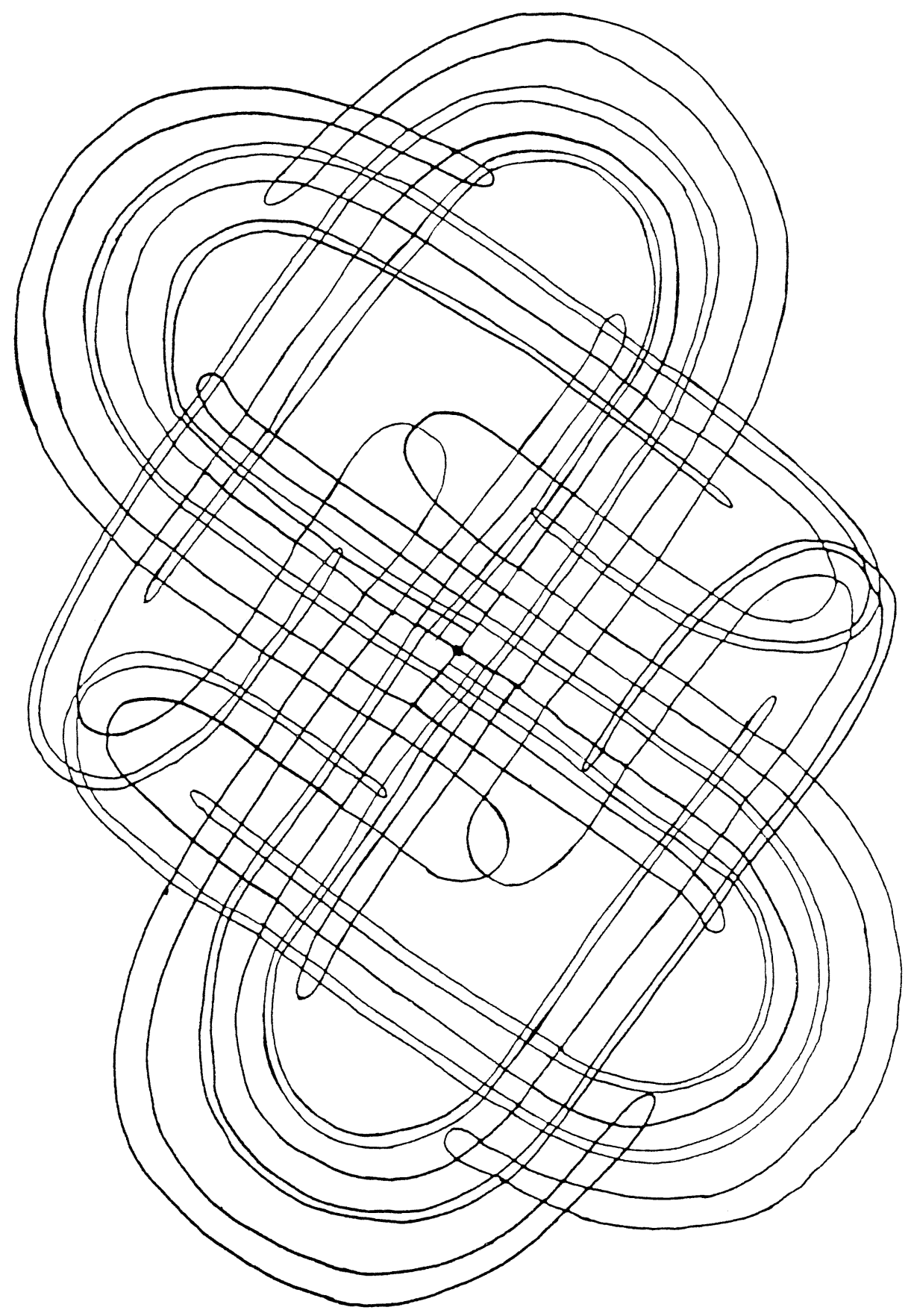

FIGURE 1 
The remainder of this paper is organized in five sections. The first section contains some definitions and results which are relevant to understanding trellises. In $\S 2$ the homoclinic trellis associated with a horseshoe map is described. In $\S 3$ some other homoclinic trellises are studied, and in $\S 4$ some model trellises are constructed. $\S 5$ contains some final remarks. As far as I know trellises have not been systematically studied and my own understanding of these beautiful objects is very incomplete.

1. Fundamental segments, stable and unstable orderings. Let $T$ be a diffeomorphism of the plane having the origin as a hyperbolic fixed point. To keep the situation as simple as possible, assume that

$$
D T(0)=\left(\begin{array}{ll}
a & 0 \\
0 & b
\end{array}\right) \quad \text { with } 0<b<1<a .
$$

Let $W^{\mathrm{s}}$ and $W^{\mathrm{u}}$ denote the stable and unstable manifolds of the origin. Let $H=$ $W^{\mathrm{s}} \cap W^{\mathrm{u}}-\{0\} . \quad H$ is the set of homoclinic points. From the stable manifold theorem one has immersions $\gamma^{\mathrm{s}}: R^{1} \rightarrow R^{2}$ and $\gamma^{\mathrm{u}}: R^{1} \rightarrow R^{2}$ with $\gamma^{\mathrm{s}}\left(R^{1}\right)=W^{\mathrm{s}}$ and $\gamma^{\mathrm{u}}\left(R^{1}\right)=W^{\mathrm{u}} ; \gamma^{\mathrm{s}}(0)=\gamma^{\mathrm{u}}(0)=(0,0) ;$ and $\dot{\gamma}^{\mathrm{u}}(0)=(1,0)$ and $\dot{\gamma}^{\mathrm{s}}(0)=(0,1)$. The trellis of the origin is the set $W^{\mathrm{s}} \cup W^{\mathrm{u}}$, and the general problem is to describe the sets $W_{ \pm}^{\mathrm{s}}, W_{ \pm}^{\mathrm{u}}, H$ and their closures, where $W_{+}^{\mathrm{s}}=\gamma^{\mathrm{s}}([0, \infty)), W_{-}^{\mathrm{s}}=\gamma^{\mathrm{s}}((-\infty, 0])$, $W_{+}^{\mathrm{u}}=\gamma^{\mathrm{u}}([0, \infty))$ and $W_{-}^{\mathrm{u}}=\gamma^{\mathrm{u}}((-\infty, 0])$.

1.1 REMARK. The trellis of the origin is a topological conjugacy invariant of the transformation $T$.

1.2 Definitions. The immersions $\gamma^{\mathrm{s}}$ and $\gamma^{\mathrm{u}}$ induce linear orderings $<_{\mathrm{s}}$ and $<_{\mathrm{u}}$ on $W^{\mathrm{s}}$ and $W^{\mathrm{u}}$ respectively. Thus $H$ has two linear orderings called its stable and unstable orderings.

For points $p, q \in H$ let $U[p, q]$ denote the closed segment of $W^{\mathrm{u}}$ with endpoints $p$ and $q$. Let $S[p, q]$ denote the closed segment of $W^{\mathrm{s}}$ with endpoints $p$ and $q$.

For $p \in H$ define the type number of $p$ to be $n(p)=\inf \left\{j \geq 0: S\left[0, T^{j}(p)\right] \cap\right.$ $U[0, p] \neq \varnothing\} . \quad n(p)$ is the number of iterates of $T$ needed to "pull" $S[0, p]$ off of the initial segment $U[0, p]$. It follows that $n(p)=n\left(T^{j}(p)\right)$ for any $j$. Hence $H$ is partitioned into sets $H_{k}=\{p \in H: n(p)=k\}$.

We call $U[p, T(p)]$ a fundamental segment provided $p \in H$ and $n(p)=0$. Similarly define a fundamental segment $S[p, T(p)]$. Now choose fundamental segments $U^{ \pm}, S^{ \pm}$with common endpoints in $W_{ \pm}^{\text {u }}$ and $W_{ \pm}^{\text {s }}$ respectively. These segments may appear as pictured in Figure 2.

The homoclinic trellis is the union of powers of $T$ applied to the segments $U^{+}, U^{-}, S^{+}, S^{-}$. For each pair of fundamental segments $S=S[p, T(p)]$ and $U=U[q, T(q)]$ there is associated an intersection matrix

$$
M(S, U)=(M(S, U, j, k))
$$

with $M(S, U, j, k)=T^{j}(S) \cap T^{k}(U)$. Each set $M(S, U, j, k)$ is ordered by the stable and unstable orderings. The matrix $M\left(S^{+}, U^{+}\right)$for example contains considerable information about $W_{+}^{\mathrm{s}} \cup W_{+}^{\mathrm{u}}$. From the definition,

$$
T^{r}(M(S, U, j, k))=M(S, U, j+r, k+r)
$$

and thus $M(S, U)$ is determined by one of its rows or one of its columns. Sometimes, as we see in $\S 3, M(S, U)$ can be computed. 


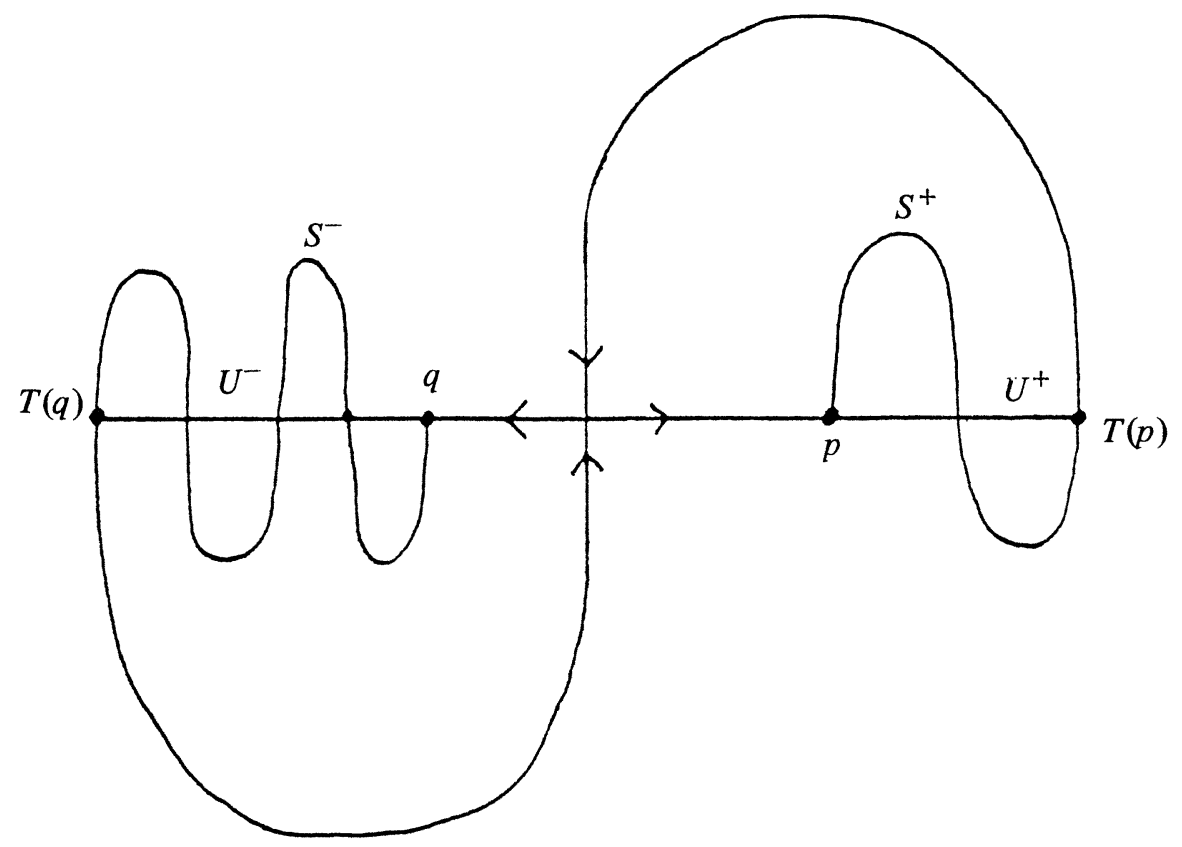

FIGURE 2

2. The trellis of a horseshoe map. An example where a homoclinic trellis can be described comes from Smale's horseshoe map. Let $B$ denote the region in the plane consisting of the unit square $[0,1] \times[0,1]$ plus two half disks $D_{0}=\{(x, y): x \leq$ $\left.0, x^{2}+(y-1 / 2)^{2} \leq 1\right\}$ and $D_{1}=\left\{(x, y): x \geq 1,(x-1)^{2}+(y-1 / 2)^{2} \leq 1\right\}$.

Suppose that $T$ is a diffeomorphism of $R^{2}$ which maps $B$ into itself as pictured in Figure 3. In particular we assume that $T$ maps two "vertical strips" $R_{0}$ and $R_{1}$ in the unit square onto two "horizontal strips" crossing the unit square as pictured.

Smale showed [3] that the invariant set $\Lambda=\left\{p \in R_{0} \cup R_{1}: T^{n}(p) \in R_{0} \cup R_{1}\right.$ for all $n\}$ is a Cantor set with hyperbolic structure and that $T$ restricted to $\Lambda$ is topologically equivalent to the shift automorphism on two symbols. A nice exposition of these results due to Conley is found in [2], and we will use his terminology. Thus a horizontal curve is the graph of a function $y=u(x)$ with $0 \leq x \leq 1,0 \leq u(x) \leq 1$ and $u^{\prime}(x) \leq 1 / 2$ (the factor $1 / 2$ could be replaced by any constant less than one). A horizontal strip is a set $U$ of the form

$$
U=\left\{(x, y): 0 \leq x \leq 1 \text { and } u_{1}(x) \leq y \leq u_{2}(x)\right\}
$$

where $y=u_{1}(x)$ and $y=u_{2}(x)$ are disjoint horizontal curves. The diameter $d(U)$ is defined to be the maximum of $\left|\left(u_{1}(x)-u_{2}(x)\right)\right|$. Vertical curves and strips are similarly defined.

Let $Q$ denote the unit square in $R^{2}$. We require that there exist $0<\lambda<1$ and vertical strips $R_{0}$ and $R_{1}$ such that

(A) $T\left(R_{0}\right)$ and $T\left(R_{1}\right)$ are horizontal strips.

(B) If $V$ is a vertical strip in $R_{0} \cup R_{1}$, then $V_{a} \equiv T^{-1}(V) \cap R_{a}$ is a vertical strip with $d\left(V_{a}\right) \leq \lambda d(V)$.

(C) If $U$ is a horizontal strip, then $U_{a} \equiv T\left(U \cap\left(R_{0} \cup R_{1}\right)\right) \cap T\left(R_{a}\right)$ is a horizontal strip with $d\left(U_{a}\right) \leq \lambda d(U)$. 

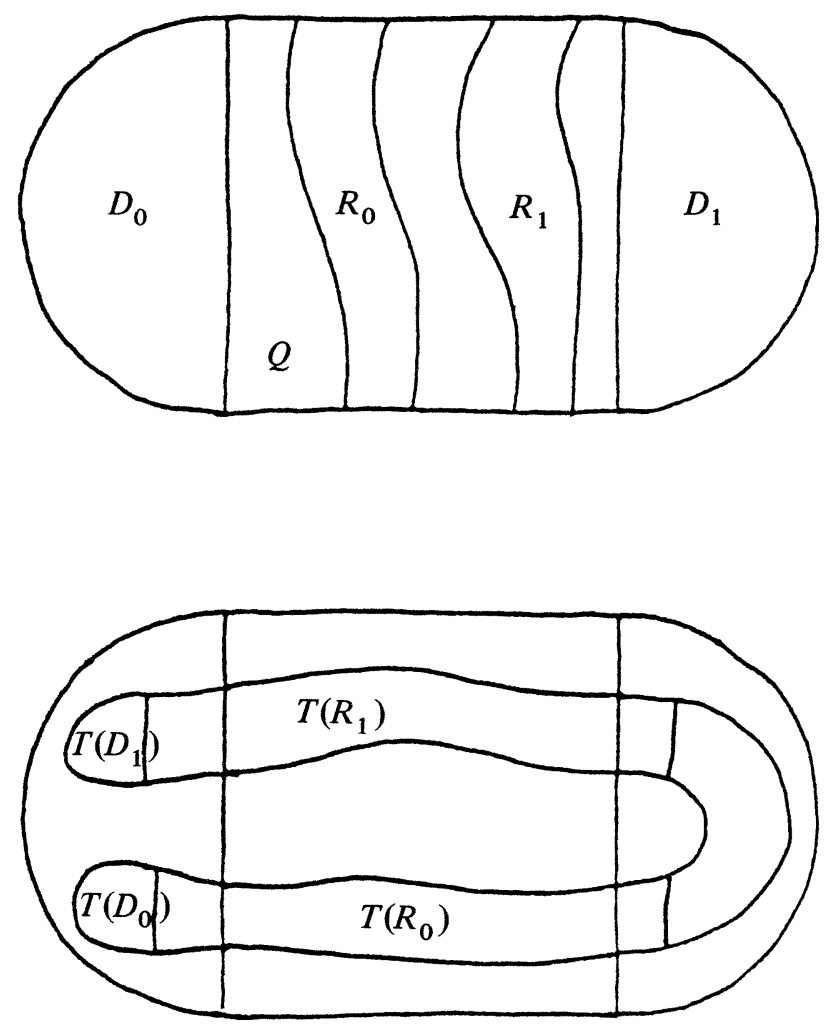

FIGURE 3

Under these conditions $T$ restricted to $\Lambda$ is topologically equivalent to a shift automorphism. The equivalence is obtained as follows: For $p \in R_{0} \cup R_{1}$ define

$$
\begin{aligned}
& n^{+}(p)=\sup \left\{k: T^{j}(p) \in R_{0} \cup R_{1} \text { for } 0 \leq j \leq k\right\}, \\
& n^{-}(p)=\inf \left\{k: T^{j}(p) \in R_{0} \cup R_{1} \text { for } k \leq j \leq 0\right\} .
\end{aligned}
$$

For $n^{-}(p) \leq j \leq n^{+}(p)$ define

$$
s(p, j)= \begin{cases}0 & \text { if } T^{j}(p) \in R_{0} \\ 1 & \text { if } T^{j}(p) \in R_{1}\end{cases}
$$

Note that if $p \in \Lambda$, then $n^{-}(p)=-\infty$ and $n^{+}(p)=+\infty$. Define $S$ to be the set of functions from the integers to the set consisting of 0 and $1 . S$ is called the set of symbols and is given the compact open topology.

The shift automorphism $\sigma: S \rightarrow S$ is defined by $(\sigma s)(j)=s(j-1)$. The topological equivalence between $T$ restricted to $\Lambda$ and $\sigma$ is by $h: \Lambda \rightarrow S$, where $h(p)(j)=s(p, j)$.

For $s \in S$ and $n>0$ define

$$
\begin{aligned}
& V(s, n)=\left\{p: s_{j}(p)=s(j) \text { for } 0 \leq j \leq n\right\}, \\
& U(s,-n)=\left\{p: s_{j}(p)=s(j) \text { for }-n \leq j \leq 0\right\} .
\end{aligned}
$$

It is shown in [2] that $V(s, n)$ and $U(s,-n)$ are vertical and horizontal strips respectively whose diameters are less than $\lambda^{n}$. The fact that $h$ is a topological equivalence 


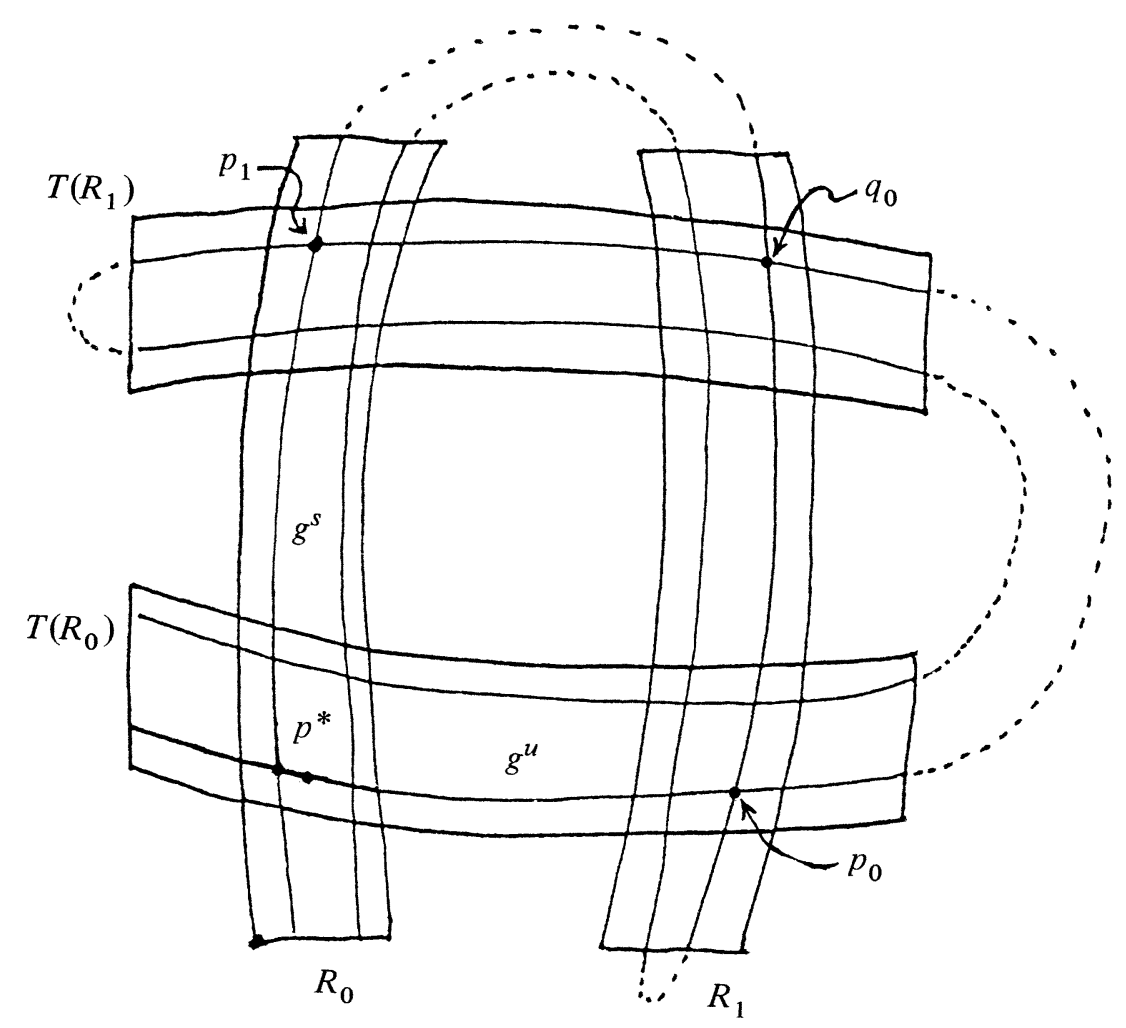

FIGURE 4

is a consequence of the result that thin vertical and horizontal strips intersect in a nonempty set of small diameter.

The horseshoe map $T$ and its relation to the shift automorphism $\sigma$ are well known to researchers in dynamical systems, but as far as I know the following discussion of its relationship with a trellis is new.

There is a unique hyperbolic fixed point $p^{*}$ of $T$ belonging to the set $R_{0} \cap T\left(R_{0}\right)$. The local unstable manifold of $p^{*}$ is a horizontal curve $g^{\mathrm{u}}$ in $T\left(R_{0}\right)$ and the local stable manifold of $p^{*}$ is a vertical curve $g^{\mathrm{s}}$ contained in $R_{0} . T\left(g^{\mathrm{u}} \cap R_{1}\right)$ is a horizontal curve in $T\left(R_{1}\right)$ which intersects $g^{\mathrm{s}}$ in a unique point $p_{1} . p_{1}$ is a transverse homoclinic point, and the homoclinic trellis of $p^{*}$ is the object of our study. Initial segments of the homoclinic trellis of $p^{*}$ are pictured in Figure 4.

For each $s \in S$ observe that each horizontal strip $U(s, n)$ contains a horizontal curve which is contained in the unstable manifold $W^{\mathrm{u}}$ of $p^{*}$. Likewise each vertical strip $V(s, n)$ contains a vertical curve which is contained in the stable manifold $W^{s}$ of $p^{*}$. Thus $U(s, n) \cap V(s, n)$ contains a point homoclinic to $p^{*}$ and consequently we have established

2.1 Proposition. $\Lambda$ is contained in the closure of $H$, where $H$ is the set of points homoclinic to $p^{*}$.

Since $T(B) \subset B$, there is an attractor $A=\bigcap\left\{T^{n}(B): n \geq 0\right\}$ contained in $B$.

2.2 Proposition. $A$ is equal to the closure of the unstable manifold of $p^{*}$. 


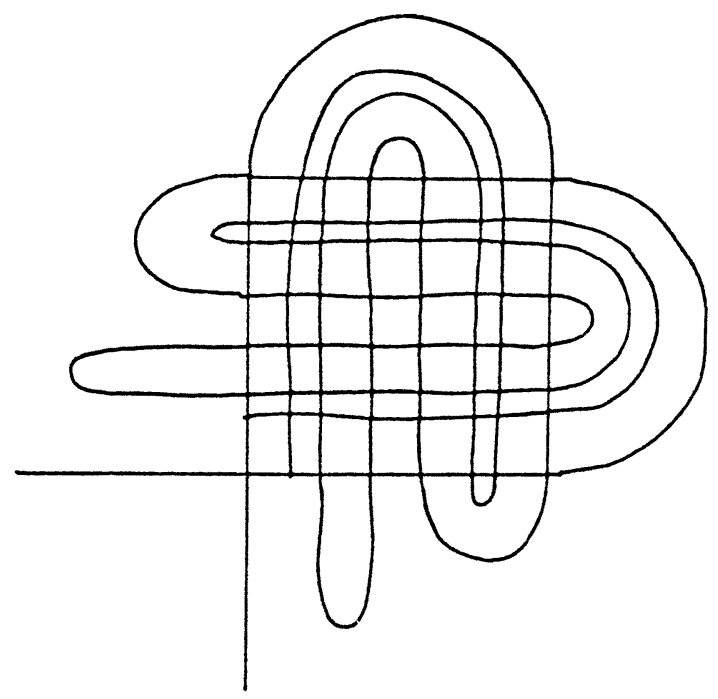

FIGURE 5

PROOF. Given $\varepsilon>0$ we will show that there exist integers $N$ and $K$ such that $T^{N+K}(B)$ is within an $\varepsilon$ neighborhood of the unstable manifold $W^{\mathrm{u}}$ of $p^{*}$. For any $x \notin R_{0} \cup R_{1}, T^{2}(x) \in D_{0} . T\left(D_{0}\right) \subset D_{0}$ and $T$ restricted to $D_{0}$ is a contraction map. Thus given $\varepsilon>0$ one can choose an integer $N>0$ such that $x \notin R_{0} \cup R_{1}$ implies $T^{n}(x)$ is within distance $\varepsilon$ from the sink in $D_{0}$ for all $n \geq N$ and hence is within $\varepsilon$ of $W^{\mathrm{u}}$.

Choose a foliation of $R_{0} \cup R_{1}$ with fibres vertical arcs such that each $x \in R_{0} \cup$ $R_{1}$ belongs to a fibre $\gamma(x)$ intersecting $g^{\text {u }}$ in a unique point $y . \quad T: T^{r}(\gamma(x)) \rightarrow$ $T^{r+1}(\gamma(x))$ contracts points by a factor of $\lambda<1$ as long as $x, T(x) \cdots T^{K}(x) \in$ $R_{0} \cup R_{1}$. Choose $K$ so that $\lambda^{K}<\varepsilon$. Thus $T^{K}(\gamma(x))$ has diameter $\leq \varepsilon$. Now consider $T^{N+K}(x)$. If $T^{N+K}(x)$ is not within $\varepsilon$ of the sink, then $T^{j}(x) \in R_{0} \cup R_{1}$ for $0 \leq j \leq K$. Hence $T^{K}(x)$ is within $\varepsilon$ of $T^{K}(y)$, with $y=\gamma(x) \cap W^{\mathrm{u}}$. It follows that $T^{N+\bar{K}}(B)$ is within $\varepsilon$ of the closure of $W^{\mathrm{u}}$. Since $\varepsilon$ is arbitrary, $A$ equals the closure of $W^{\mathrm{u}}$.

A model for the trellis of a horeshoe map can be constructed as follows: The "middle third" Cantor set $C$ consists of points in the unit interval that can be represented in the form $x=\sum_{k=1}^{\infty} a_{k} 3^{-k}$, with $a_{k} \in\{0,2\}$. An endpoint of $C$ is a point $x \in C$ such that for some $n, a_{k}=a_{n}$ whenever $k \geq n$. Let $E$ denote the set of endpoints of $C$. Let $A$ and $B$ denote the linear transformations of $R^{2}$ defined by $A(x, y)=(3 x, 1 / 3 y)$ and $B(x, y)=(y, x)$. The model trellis $T$ consists of $\left\{T_{j} \cup T_{j}^{\prime}: j \geq 0\right\}$ where $T_{j}^{\prime}=B\left(T_{j}\right)$, and where

$$
\begin{aligned}
& T_{0}=\{(x, y): 0 \leq x \leq 1, y \in E\}, \\
& T_{1}=\left\{(x, y): x \geq 1,(x-1)^{2}+(y-1 / 2)^{2}=(1 / 2-e)^{2} \text { for some } e \in E\right\} \\
& T_{2}=\left\{(x, y): x \leq 1, x^{2}+(y-5 / 6)^{2}=(5 / 6-e)^{2} \text { for some } e \in E \cap[2 / 3,1]\right\}, \\
& T_{j+1}=A\left(T_{j}\right) \text { for } j \geq 2 .
\end{aligned}
$$

A beginning development of the model trellis is pictured in Figure 5 . 


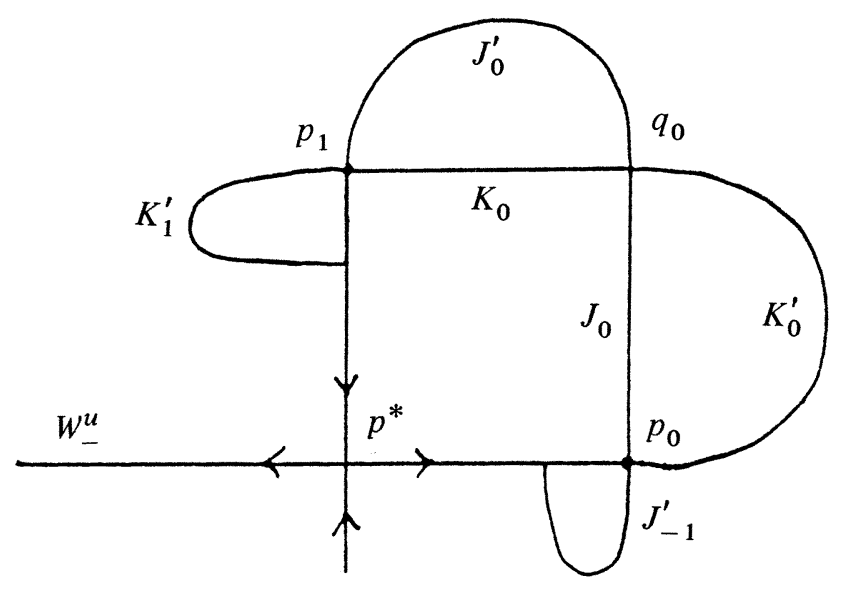

FIGURE 6

It is natural to ask: In what sense is the model trellis "equivalent" to the trellis of a horseshoe map? Several definitions are possible.

2.3 DEFinition. We say that two trellises are strongly equivalent if there exists a homeomorphism of the plane taking one onto the other. They are equivalent if their closures are homeomorphic.

A subtrellis $\tau_{n}$ of a trellis $\tau$ is a subset of the form $\tau_{n}=S\left[a_{n}, b_{n}\right] \cup U\left[c_{n}, d_{n}\right]$. A development of the trellis $\tau$ is a sequence of subtrellises $\left\{T_{n}\right\}$ such that $\tau_{0}=p^{*}$, $\tau_{n} \subset \tau_{n+1}$ for each $n \geq 0$, and $\bigcup\left\{\tau_{n}: n \geq 0\right\}=\tau$. Trellises $\tau$ and $\tau^{\prime}$ are weakly equivalent if they have developments $\left\{\tau_{n}\right\}$ and $\left\{\tau_{n}^{\prime}\right\}$ such that, for each $n, \tau_{n}$ is homeomorphic to $\tau_{n}^{\prime}$ with a homeomorphism which preserves the respective stable and unstable orderings.

In the next section we will begin an investigation of weak equivalence. Any two horseshoe trellises will be shown to be weakly equivalent (see Theorem 3.5). However, it is probably true that they are equivalent and even strongly equivalent. In this direction Marcy Barge [2] has shown that any two horseshoe attractors are homeomorphic. This result is satisfying to me and takes away some of the motivation for the technical work necessary to establish equivalence of horseshoe trellises.

3. Trellises of type $l$. The goal here is to describe the formation of a homoclinic trellis in a sequence of steps. In particular we suppose that $p^{*}$ is a hyperbolic fixed point having initial segments of stable and unstable manifolds imbedded in $R^{2}$ as pictured in Figure 6. Thus the "stage 0" construction of the trellis is the same as for the horseshoe map. Important segments of the stable and unstable manifolds are defined by

$$
J_{0}=S\left[q_{0}, p_{0}\right], \quad J_{0}^{\prime}=S\left(p_{1}, q_{0}\right), \quad K_{0}=U\left[q_{0}, p_{1}\right], \quad \text { and } \quad K_{0}^{\prime}=U\left(p_{0}, q_{0}\right) .
$$

Also define $J_{n}=T^{n}\left(J_{0}\right), J_{n}^{\prime}=T^{n}\left(J_{0}^{\prime}\right)$, etc. Notice that $W_{+}^{\mathrm{u}}=\bigcup\left\{K_{n} \cup K_{n}^{\prime}\right\}$ and $W_{+}^{\text {s }}=\bigcup\left\{J_{n} \cup J_{n}^{\prime}\right\}$.

In order to keep the trellis as simple as possible we assume the following. 
Hypothesis A. The sequence $\left\{K_{n}^{\prime}\right\}$ and $\left\{J_{-n}^{\prime}\right\}$ are sequences of semicircular arcs which accumulate along $W_{-}^{\mathrm{u}}$ and $W_{-}^{\mathrm{s}}$ respectively. We also assume that for each $n \geq 0, J_{-n}^{\prime}$ and $K_{n}^{\prime}$ contain no homoclinic points.

In order to describe the trellis formed by the stable and unstable manifolds of $p^{*}$ it is necessary to impose an additional hypothesis.

3.1 Definition. $q \in H \cap K_{0}$ has transition number $t$ if $T^{t}(q) \in J_{0} . t(q)$ will denote the transition number of $q$ and $h_{n}$ will denote the subset of $H \cap K_{0}$ of points having transition number less than or equal to $n$.

\subsection{LEMMA. $q$ has transition number $n$ if and only if $q$ has type number $n$.}

Proof. If $q$ has type number $n$, then $S\left[0, T^{n}(q)\right] \cap U[0, q] \neq \varnothing$. Hence $p_{1}<_{\mathrm{s}}$ $T^{n}(q)$. Also, $S\left[0, T^{n+1}(q)\right] \cap U(0, q]=\varnothing$, and it follows that $T^{n+1}(q)<_{\mathrm{s}} p_{1}$. Therefore $T^{n}(q) \in J_{0}$ so $t(q)=n$.

If $t(q)=n$, then $S\left[0, T^{n}(q)\right] \cap U[0, p] \neq \varnothing$ because $T^{n}(q) \in J_{0}$. Further, $T^{n+1}(q) \in J_{1}$ and hence $S\left[0, T^{n+1}(q)\right] \cap U[0, q]=\varnothing$. Therefore $q$ has type number $n$.

Hypothesis $\mathrm{B}$. There exists a positive integer $l$ such that

(1) if $a, b$ are $<_{u}$ adjacent points of $h_{n}$ with $t(a)=t(b)$, then $T^{n}(U[a, b])$ is contained in the $D$-shaped region bounded by $J_{r} \cup K_{r}^{\prime}$, where $r=n-t(a)$.

(2) If $a, b$ are $<_{u}$ adjacent points of $h_{n}$ with $t(a)<t(b)$, then $T^{n}(U[a, b]) \cap J_{-1}$ contains exactly two points whenever $n-t(a) \geq l$ and is empty whenever $n-t(a)<l$.

3.3 Definition. A trellis which satisfies Hypotheses A and B is called a trellis of type $l$.

\subsection{Proposition. A horseshoe trellis is a trellis of type one.}

PROOF. In the horseshoe trellis, $J_{-1}$ intersects the square $Q$ in two vertical arcs as is shown in Figure 4. Further $T^{n}(U[a, b])$ is a horizontal arc crossing $Q$ and thus $T^{n}(U[a, b])$ intersects $J_{-1}$ in exactly two points. Hypothesis A is evidently also satisfied.

The beginning development of a trellis of type 2 is pictured in Figure 7 .

We can now state the main result of this section.

\subsection{THEOREM. Any two trellises of type $l$ are weakly equivalent.}

ProOF. Let $\tau$ and $* \tau$ denote two trellises of type $l$. We assume that $\tau$ and * $T$ consist respectively of the stable and unstable manifolds of the same saddle point $p^{*}$ with respect to diffeomorphisms $T$ and ${ }^{*} T$ of the plane. Since they are trellises of type $l$, we may also assume that the subtrellises $\tau_{1}$ and ${ }^{*} \tau_{1}$ coincide where $\tau_{1}=W_{-}^{\mathrm{u}} \cup W_{-}^{\mathrm{s}} \cup U\left[p^{*}, p_{1}\right] \cup S\left[p^{*}, p_{0}\right]$ (see Figure 6). Let $\tau_{n}=W_{-}^{\mathrm{u}} \cup$ $W_{-}^{\mathrm{s}} \cup U\left[p^{*}, p_{n}\right] \cup S\left[p^{*}, p_{-n+1}\right]$. Thus $\left\{\tau_{n}\right\}$ forms a development of $\tau$ and $\left\{{ }^{*} \tau_{n}\right\}$ forms a development of ${ }^{*} T$, where ${ }^{*} \tau_{n}=W_{-}^{\mathrm{u}} \cup W_{-}^{\mathrm{s}} \cup U\left[p^{*},{ }^{*} p_{n}\right] \cup S\left[p^{*},{ }^{*} p_{n-1}\right]$ and ${ }^{*} p_{j}={ }^{*} T^{j}\left(p_{0}\right)$. Note that an object 0 associated with the trellis $\tau$ will correspond with an object ${ }^{*} 0$ in the trellis ${ }^{*} \tau$ in our notation. Thus ${ }^{*} H$ will denote the set of homoclinic points in $* \tau$.

We pause here in the proof to establish a lemma which is needed to construct a homeomorphism of the subtrellis $\tau_{n}$ onto the subtrellis ${ }^{*} \tau_{n}$. 


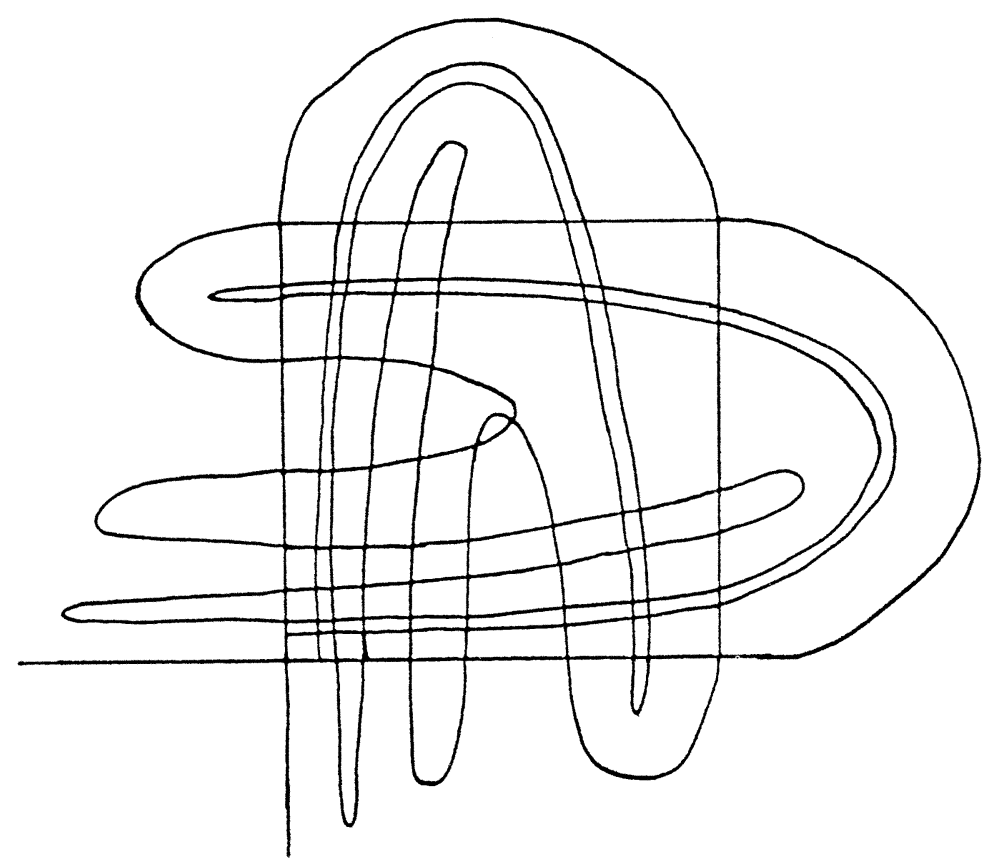

FIGURE 7

3.6 LEMMA. There exists a one-to-one correspondence $g: H \cap K_{0} \rightarrow{ }^{*} H \cap K_{0}$ which preserves the stable and unstable orderings and type numbers.

Proof. Start by defining $g\left(q_{0}\right)=q_{0}$ and $g\left(p_{1}\right)=p_{1}$. Inductively define $g$ by assuming that $g: h_{n} \rightarrow{ }^{*} h_{n}$ has been defined and is a one-to-one correspondence which preserves the stable and unstable orderings.

Let $a$ and $b$ denote a pair of $<_{\mathrm{u}}$ adjacent points of $h_{n}$ with $U[a, b] \cap h_{n+1} \neq \varnothing$ and $t(a)<t(b)$. By Hypothesis B there exist exactly two points $x, y \in h_{n+1} \cap U[a, b]$. Choose the notation so that $x$ is between $a$ and $y$ according to the unstable ordering. There also exist exactly two points $x^{\prime}, y^{\prime} \in{ }^{*} h_{n+1} \cap U[g(a), g(b)]$. Extend the definition of $g$ to map $\left\{x^{\prime}, y^{\prime}\right\}$ preserving the unstable order. This definition clearly extends $g$ as a one-to-one correspondence between $h_{n+1}$ and ${ }^{*} h_{n+1}$ which preserves the unstable ordering.

To show that $g$ preserves the stable order we refer to Figure 8 . This figure pictures part of the trellis $T$. From Hypothesis B, $J_{0}$ contains exactly two type $l$ points $\alpha, \beta$ with $\beta<_{\mathrm{s}} \alpha$. Consistent with our previous notation we let $\alpha_{-1}=$ $T^{-1}(\alpha)$ and $\beta_{-1}=T^{-1}(\beta)$. Since $t(a)<t(b)$ and $U[a, b] \cap h_{n+1}=\varnothing$ it follows from Hypothesis B that $n-t(a) \geq l$. Therefore $T^{n}(a) \in S\left[p^{*}, p_{l}\right]$.

From Figure $8, T^{n}(y)<_{\mathrm{s}} T^{n}(x)$. There is a similar figure occurring in the trellis ${ }^{*} \tau$ and therefore we also have $\left({ }^{*} T\right)^{n}\left(y^{\prime}\right)<\left({ }^{*} T\right)^{n}\left(x^{\prime}\right)$ establishing that the extension of $g$ preserves the stable orderings. This completes the proof of the lemma.

Returning to the proof of the theorem we extend the function $g$ from Lemma 3.6 from $H \cap K_{0}$ to $H$ as follows. If $T^{K}(x) \in H \cap K_{0}$, define $g(x)=\left({ }^{*} T\right)^{-K} g\left(T^{K}(x)\right)$. Clearly $g$ preserves the stable and unstable orderings. 


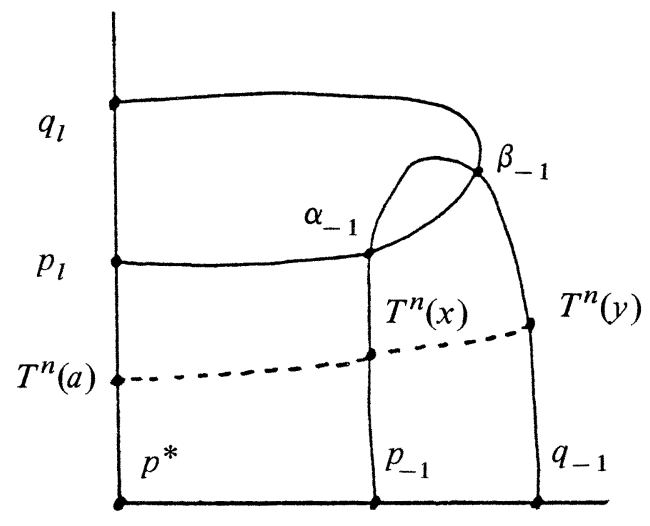

FIGURE 8

Now suppose $U[x, y]$ is a segment of the trellis $\tau$ with $U[x, y] \cap H=\{x, y\}$. Extend $g$ from $U[x, y]$ to $U[g(x), g(y)]$ so that the ratio of the arc length of $U[x, z]$ to the arc length of $U[g(x), g(z)]$ is constant as a function of $z$. Thus we say that $g$ preserves proportional arc lengths.

Similarly extend $g$ to a segment $S[x, y]$ of the trellis $\tau$. Note that with this definition the restriction of $g$ from the partial trellis $\tau_{n}$ to the partial trellis ${ }^{*} \tau_{n}$ is a homeomorphism and therefore the trellises $\tau$ and $\tau^{\prime}$ are weakly equivalent. This completes the proof.

There is a difficulty in showing that two weakly equivalent type $l$ trellises are equivalent. Suppose that $x_{0} \in \mathcal{T}$ and $x_{j} \rightarrow x_{0}$ as $j \rightarrow \infty$. If the sequence $\left\{x_{j}\right\}$ is not contained in any partial development $\tau_{n}$ of the trellis $\tau$, then it is perhaps possible that the sequence $\left\{g\left(x_{j}\right)\right\}$ does not converge to $g\left(x_{0}\right)$.

Now suppose that the trellis of $p^{*}$ satisfies Hypotheses A and B. We will show that the integer $l$ in Hypothesis B determines the combinatorial structure of the trellis and that different $l$ 's produce different trellises. Consider the fundamental segments $S=S\left(P_{1}, P_{0}\right]=J_{0}^{\prime} \cup J_{0}$ and $U=U\left(P_{0}, P_{1}\right]=K_{0}^{\prime} \cup K_{0}$. Recall that in $\S 1$ an intersection matrix $M(S, U)$ associated with these segments was defined. For $i, j \geq 0$ let $M_{i, j}=T^{i}(S) \cap T^{j}(U)$ and let $M$ denote the matrix with entries $M_{i, j}$. By Hypothesis A we have $J_{i}^{\prime} \cap K_{j}^{\prime}=\varnothing$ for all $i$ and $j$. Therefore $M_{i, j}=J_{i} \cap K_{j}$ and also $M_{i+k, j+k}=T^{k}\left(M_{i, j}\right)$.

Briefly, the reason that the trellis is determined by $l$ is because $l$ determines the intersection matrix $M$. The $n$th column of $M$ is formed by the intersection of $K_{n}$ with $J_{0}, J_{1}, J_{2}$, and so on. From Hypothesis B there exist injective functions $\gamma_{0}, \gamma_{1}: \bigcup_{i=l}^{\infty} J_{i} \cap H \rightarrow J_{-1} \cap K_{n}$ such that $J_{-1} \cap K_{n}$ is the disjoint union of the ranges of $\gamma_{0}$ and $\gamma_{1}$. Consequently $M_{0, n+1}=T\left(J_{-1} \cap K_{n}\right)$ is determined by the $n$th column of $M$. Since $M_{i, n+1}=T\left(M_{i-1, n}\right)$ the $(n+1)$ th column of $M$ is determined by the $n$th column of $M$ and therefore $M$ is entirely determined by its zeroth column. But $M_{0,0}=\left\{q_{0}\right\}, M_{0,1}=\left\{P_{1}\right\}$ and $M_{0, j}=\varnothing$ for $j>1$. Hence the zeroth column of $M$ is known.

Let $r_{i, j}$ denote the number of points in $M_{i, j}$ and let $R$ denote the matrix with 
entries $r_{i, j}$. Then for $l=2$ the matrix $R$ looks as follows:

$$
R=\left[\begin{array}{lllllll}
1 & 0 & 2 & 4 & 4 & 8 & \\
1 & 1 & 0 & 2 & 4 & 4 & \\
0 & 1 & 1 & 0 & 2 & 4 & \\
0 & 0 & 1 & 1 & 0 & 2 & \ldots \\
0 & 0 & 0 & 1 & 1 & 0 & \\
0 & 0 & 0 & 0 & 1 & 1 & \\
0 & 0 & 0 & 0 & 0 & 1 &
\end{array}\right]
$$

In particular, $r_{0, n}=2\left(\sum_{j=0}^{n-2} r_{0, j}+1\right)$ and the sequence $\left\{r_{0, n}\right\}$ is something like a Fibonnaci sequence. For general $l$ we have the relation $r_{0, n}=2\left(\sum_{j=0}^{n-l} r_{0, j}+1\right)$ and this shows the intersection matrices for different $l$ 's are distinct.

4. Model of type $l$ trellises. At the end of $\S 2$ a model for a horseshoe trellis was constructed based on joining the endpoints of a middle third Cantor set in a certain way. Examples of this sort were considered by topologists for other purposes and are known as Knaster continua.

The model for the type $l$ trellis will be described in terms of its development. The unstable manifold $W_{+}^{\mathrm{u}}\left(p^{*}\right)$ weaves across the square in a certain pattern (see Figure 7). This pattern is determined by the set $h_{n}$ of homoclinic points in $K_{0}$ with type number less than or equal to $n$. From $h_{n}$ the set $h_{n+1}$ is determined according to Hypothesis B as follows: If $a$ adn $b$ are two $<_{\mathbf{u}}$ adjacent points of $h_{n}$ with $t(a)<t(b)$, then $U[a, b] \cap h_{n+1}$ contains exactly two points if and only if $n-l \geq t(a) . U[a, b] \cap h_{n+1}$ is empty when $t(a)=t(b)$ or $n-l<t(a)$.

One can use this algorithm to construct the sets $h_{n}$ as subsets of the endpoints of the standard middle third Cantor set. Note that this gives a "strange" construction of the Cantor set. The set $h_{4}$ is pictured below with the type number of each point written above it.

\begin{tabular}{cccccccccccc}
0 & 4 & 4 & 3 & 3 & 2 & 2 & 3 & 3 & 4 & 4 & 0 \\
& 1 & 1 & $\perp$ & 1 & 1 & 1 & 1 & $\perp$ & 1 & & \\
\hline
\end{tabular}

Now we are ready to construct a model trellis of type $l$. We concentrate on describing the development of $W_{+}^{\mathrm{u}}$. $W_{+}^{\mathrm{u}}$ consists of the initial segment $U\left[p^{*}, p_{1}\right]$ together with the segments $K_{n}^{\prime} \cup K_{n}$ for $n \geq 1$. By Hypothesis A, $K_{n}^{\prime}$ is a semicircular arc close to $W_{-}^{\mathrm{u}}$ which joins $q_{n}$ and $p_{n}$ (see Figure 7). However, the segment $K_{n}$ must weave through the segments $J_{0}, \ldots, J_{n}$ in a complicated way.

4.1. Define the weaving set $W_{n}$ of $K_{n}$ to be $K_{n} \cap S\left[p^{*}, p_{0}\right]$. By Hypothesis A, $K_{n} \cap J_{1}^{\prime}=\varnothing$ for all $i$, and therefore $W_{n}=\bigcup_{i=1}^{\infty} M_{i, n}$. In other words the weaving set is the union of the sets forming the $n$th column of the intersection matrix $M$. The embedding of $K_{n}$ in the plane is essentially determined by the requirements that $K_{n} \cap U\left[p^{*}, p_{n}\right]=p_{n}$ and that $K_{n}$ runs through the weaving set in the order determined by the unstable ordering of these points.

Note that $K_{n} \cap J_{0}$ consists exactly of the homoclinic points in $J_{0}$ having type number $n$ and more generally $K_{n} \cap J_{r}$ consists of homoclinic points of type number $n-r$. Thus suppose that a model trellis of type $l$ has been partly constructed and consists of $W_{-}^{\mathrm{u}} \cup W_{+}^{\mathrm{u}} \cup S\left[p^{*}, q_{0}\right] \cup U\left[p^{*}, p\right]$. At this stage we add $K_{n}^{\prime}$ as a semicircular loop joining $q_{n+1}$ and $p_{n+1}$ and folded close to $W_{-}^{\mathrm{u}}$. To each of the segments $J_{r}$ for 


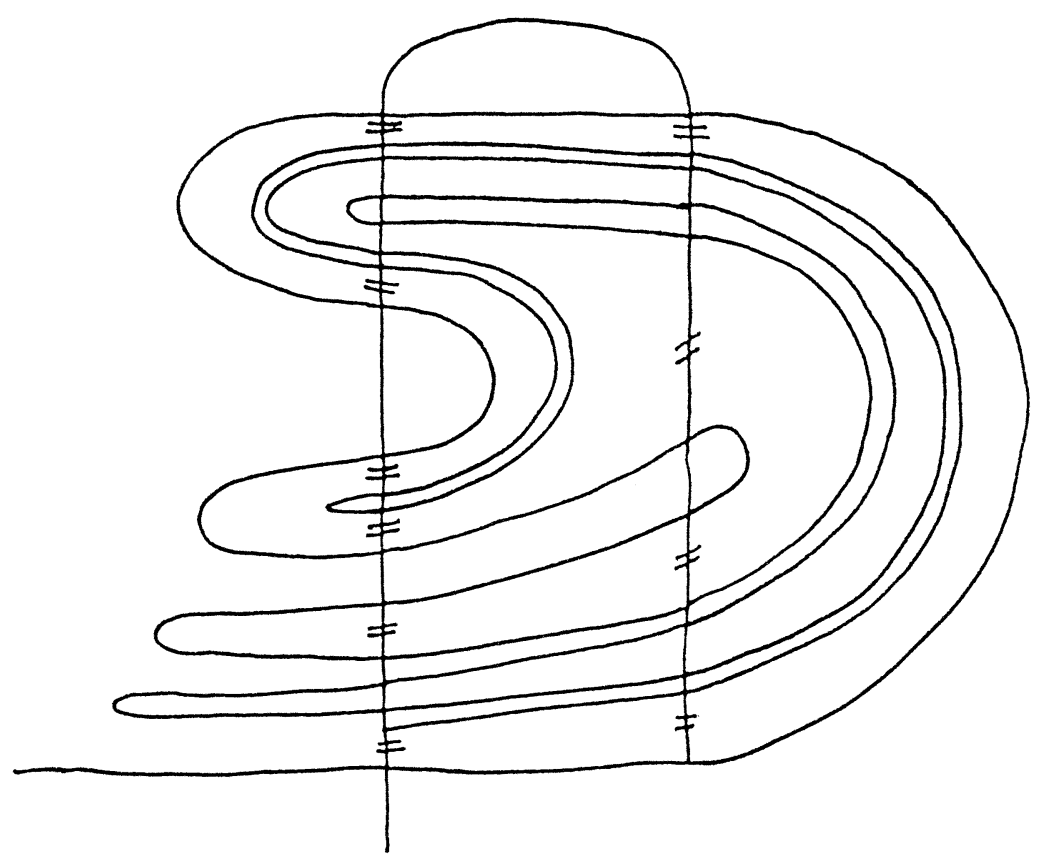

FIGURE 9

$0 \leq r \leq n+1$ add type $n-r$ endpoints according to the "strange" construction of a middle third Cantor set in $J_{r}$. In the $D$ shaped region bounded by $J_{r} \cap K_{r}^{\prime}$ symmetrically join the upper and lower type $n-r$ endpoints with semicircular arcs which do not intersect the previously constructed initial segment $U\left[p^{*}, p_{n}\right]$ of the unstable manifold.

Next join all points of the weaving set $W_{n}$ with arcs inside the square $Q$ which do not intersect $U\left[p^{*}, p_{n}\right]$. The important point here is that there is exactly one way to do this. All the new arcs connect to form $K_{n}$ and are added to the development of $W_{+}^{n}$. Figure 9 above indicates the construction for $l=2$ and $n=5$. Type $5-r$ points have been added to $J_{r}$ for $0 \leq r \leq 5$ but the connecting arcs constituting $K_{5}$ have not been drawn. In analogous fashion one constructs $W_{+}^{\text {s }}$ consisting of $S\left[p^{*}, p_{0}\right]$ and the $\operatorname{arcs} J_{-n}^{\prime} \cup J_{-n}$ for $n \geq 1$. The construction is determined by requiring $S\left[p^{*}, p_{n}\right]$ to cross $U\left(p^{*}, p_{n}\right]$ transversally a minimum number of times.

5. Conclusion. The construction of a model type $l$-trellis was not precise enough to determine its closure and indeed it is possible for trellises of type $l$ to have topologically inequivalent closures. Describing the combinatorics of a trellis such as the one pictured in Figure 1 presents a challenge. Perhaps this paper has made a start in that direction. I do not expect the combinatorics of that trellis to be determined at any finite stage of its development. Ultimately one might hope to understand models of trellises as in Figure 1 and to construct models whose closures will give concrete examples of ergodic zones. 


\section{REFERENCES}

1. R. Adler and B. Weiss, Similarity of automorphisms of the torus, Mem. Amer. Math. Soc., no. 98 (1970).

2. M. Barge, Horseshoe maps and inverse limits (to appear).

3. J. Moser, Stable and random motions in dynamical systems, Ann. of Math. Studies, no. 77, Princeton Univ. Press, Princeton, N.J., 1973.

4. S. Smale, Diffeomorphisms with many periodic points, Differential and Combinatorial Topology (S. S. Cairns, Ed.), Princeton Univ. Press, Princeton, N.J., 1965, pp. 267-292.

Department of Mathematics, University of Colorado, Boulder, Colorado 80309 